\title{
Biometria de sementes de Byrsonima verbascifolia (L.) DC. provenientes da região semiárida da Bahia
}

\author{
Janisson Batista de Jesus ${ }^{1 *}$, José Monteiro do Nascimento Júnior ${ }^{2}$, Dráuzio Correia Gama ${ }^{3}$, Alisson \\ Marcel Souza de Oliveira ${ }^{2}$
}

\begin{abstract}
RESUMO: Os estudos em sementes florestais, especificamente de biometria, são essenciais para o conhecimento das espécies e podem ter aplicação para a conservação, produção de mudas, reflorestamento e melhoramento genético. $\mathrm{O}$ objetivo do presente estudo foi realizar a caracterização biométrica de sementes de Byrsonima verbascifolia procedentes do município de Euclides da Cunha, região do semiárido do estado da Bahia. As sementes foram obtidas de diferentes matrizes no município de Euclides da Cunha, Bahia, sendo obtidos os valores de comprimento, largura e espessura $(\mathrm{cm})$, peso $(\mathrm{g})$, peso de mil sementes $(\mathrm{g})$ e número de sementes por $\mathrm{kg}$. Observou-se a média de $0,83 \mathrm{~cm}$ de comprimento, $0,9 \mathrm{~cm}$ de largura, $0,88 \mathrm{~cm}$ de espessura, e massa específica de $0,66 \mathrm{~g}$, peso de mil sementes de $335,47 \mathrm{~g}$ e 2.981,26 sementes por kg. O estudo permitiu caracterizar biometricamente as sementes de Byrsonima verbascifolia, contribuindo para o conhecimento e a divulgação da espécie que é de ocorrência da região semiárida do estado da Bahia, servindo de base para ampliar novos estudos científicos.
\end{abstract}

Palavras-chave: murici, semente florestal, Caatinga

\section{Biometry of seeds of Byrsonima verbascifolia (L.) DC. from the semiarid region of the Bahia}

\begin{abstract}
Studies on forest seeds specifically of biometrics are essential for the knowledge of the species and can be used for conservation, seedling production, reforestation and breeding. The objective of the present study was to perform the biometric characterization of Byrsonima verbascifolia (L.) Rich from the municipality of Euclides da Cunha, semiarid region of the state of Bahia. The seeds were obtained from different matrices in the city of Euclides da Cunha, Bahia. The values of length, width and thickness $(\mathrm{cm})$, weight $(\mathrm{g})$, weight of one thousand seeds $(\mathrm{g})$ and number of seeds per kg. It was observed the mean of $0.83 \mathrm{~cm}$ of length, $0.9 \mathrm{~cm}$ of width, $0.88 \mathrm{~cm}$ thickness, and weight of 0.66 $\mathrm{g}$, weight of one thousand seeds of $335.47 \mathrm{~g}$ and 2.981 .26 seeds per $\mathrm{kg}$. The study allowed to characterize biometrically the seeds of Byrsonima verbascifolia, contributing to the knowledge and the divulgation of the species that is occurring in the semiarid region of the state of Bahia, serving as the basis to expand new scientific studies.
\end{abstract}

Keywords: murici, forest seed, Caatinga

\section{INTRODUÇÃO}

As pesquisas de sementes florestais nativas são fundamentais para alcançar mudas de qualidade e em quantidades que atendam aos segmentos do setor florestal (LEÃO et al., 2015), uma vez que a propagação via sementes é a principal maneira de disseminação das espécies e o estudo de tecnologia e produção de sementes assumem grande relevância no processo de manejo, conservação e melhoramento genético (ROVERI NETO e PAULA, 2017).

A análise da biometria de sementes é muito utilizada na diferenciação da qualidade fisiológica das espécies vegetais (LUCENA et al., 2017), contribuindo significativamente na distinção de espécies de mesmo gênero (ASSIS et al., 2013), constituindo um notável instrumento para detectar a variabilidade genética de uma mesma espécie dentro de uma população, bem como as relações entre esta variabilidade e os fatores ambientais (ARAÚJO et al., 2015). Além disso, serve como uma das estratégias de se uniformizar a emergência em campo das plântulas, classificando-se as sementes por tamanho ou por massa e, então, selecionando-se as sementes com maior vigor (ARAÚJO et al., 2014).

As informações relacionadas aos aspectos morfológicos de frutos, sementes, plântulas e germinação também são relevantes por servirem de subsídios para o uso das espécies florestais em planos de manejo e projetos de recuperação de áreas degradadas (SILVA et al., 2014a), principalmente quando associadas aos fatores que podem afetar a

Recebido em 23/01/2018; Aceito para publicação em 21/03/2018

${ }^{1}$ Universidade Federal do Rio Grande do Sul.

2 Universidade Federal de Sergipe.

${ }^{3}$ Universidade Estadual do Sudoeste da Bahia.

*E-mail: janisson-batista-de-jesus@ hotmail.com 
emergência, como água e temperatura (ANDRADE, et al., 2016).

Várias pesquisas sobre espécies florestais já foram desenvolvidas nos biomas brasileiros, e na Caatinga pode-se citar alguns tais como os realizados em: Dalbergia nigra (Vell.) Allemão ex Benth. (BRAZ et al., 2009), Amburana cearensis (Allemão) A.C.Sm. (LOUREIRO et al., 2013), Ziziphus joazeiro Mart. (ARAÚJO et al., 2015), Spondias tuberosa Arruda (COSTA et al., 2015), Licania rigida Benth. (DINIZ et al., 2015). A Bahia apesar de ser o maior Estado da região semiárida do país ainda apresenta poucos estudos em sementes florestais, sendo verificado em: Chloroleucon foliolosum (Benth.) G.P.Lewis (SILVA et al., 2014b) e Libidibia ferrea (Mart. ex Tul.) L.P.Queiroz (SILVA et al., 2017), e especificamente em relação à Byrsonima verbascifolia (L.) DC. ainda não foi observada produção científica que forneça conhecimento sobre a espécie neste estado.

A espécie Byrsonima verbascifolia, da família Malpighiaceae, é nativa do Brasil, tem como nome popular murici, é de vasta ocorrência nos estados do nordeste e de vários domínios fitogeográficos entre eles a Caatinga (FLORA DO BRASIL, 2018). Ela é muito utilizada pela população sertaneja, na medicina popular, além disso, o extrativismo dos seus frutos contribui como fonte de renda para as suas famílias pela comercialização em feiras livres e mercados públicos, fazendo parte da alimentação in natura ou via fabricação de sucos, doces, sorvetes e licores (GUSMÃO et al., 2006).

O conhecimento sobre as sementes florestais da Caatinga são fundamentais para a preservação desta tipologia florestal (DANTAS et al., 2014), e por isso, o objetivo do presente estudo foi realizar a caracterização biométrica de sementes de Byrsonima verbascifolia (L.) DC. procedentes do município de Euclides da Cunha, região do semiárido do estado da Bahia, a fim de divulgar informações sobre as características físicas da espécie e possibilitar um maior conhecimento a respeito das suas sementes nesta região.

\section{MATERIAL E MÉTODOS}

As sementes de Byrsonima verbascifolia (L.) DC. foram coletadas em diferentes matrizes no município Tabela 1. Valores das características biométricas $($ Méd $=$ média, Mín $=$ mínimo, Máx $=$ máximo, $\mathrm{DP}=$ desvio-padrão, $\mathrm{CV}=$ coeficiente de variação) das sementes de Byrsonima verbascifolia (L.) DC., provenientes da região semiárida da Bahia.

\begin{tabular}{cccccc}
\hline Parâmetros & Méd & Mín & Máx & DP \pm & CV(\%) \\
\hline Comprimento $(\mathrm{cm})$ & 0,83 & 0,70 & 0,97 & 0,054 & 6,51 \\
Largura $(\mathrm{cm})$ & 0,90 & 0,65 & 1,06 & 0,072 & 8,00 \\
Espessura (cm) & 0,88 & 0,65 & 1,03 & 0,069 & 7,84 \\
Peso $(\mathrm{g})$ & 0,66 & 0,16 & 0,49 & 0,070 & 10,61 \\
Peso de mil sementes $(\mathrm{g})$ & 335,47 & - & - & - & - \\
$\mathrm{N}^{\circ}$ de sementes/kg & 2981,26 & - & - & & - \\
\hline
\end{tabular}

de Euclides da Cunha, Bahia, que possui uma área de cerca de $2.325 \mathrm{~km}^{2}$, localizado entre as coordenadas UTM 24 S, datum SIRGAS 2000: $466532 / 8870675$ e 567095/8817868 e tendo quase todo seu território situado na porção norte da bacia hidrográfica do rio Itapicuru. O município está enquadrado climaticamente na região semiárida, com chuvas anuais inferiores a $700 \mathrm{~mm}$, apresentando uma cobertura vegetal com predomínio de pastagens entremeadas por áreas com vegetação de Caatinga (INEMA, 2017).

Para a caracterização da biometria das sementes da espécie estudada foi utilizado aleatoriamente 100 amostras de sementes, sendo cada uma analisada individualmente, de um lote formado por 15 matrizes. Com o auxílio de um paquímetro digital $(0,01 \mathrm{~mm})$ da marca Digimess YT202, obteve-se os valores de comprimento, medindo as sementes, longitudinalmente, sentido base-hilo. E a largura e a espessura medidos, perpendicularmente, ao sentido base-hilo, considerando os valores obtidos em centímetro $(\mathrm{cm})$. As avaliações de peso $(\mathrm{g})$, peso de mil sementes $(\mathrm{g})$ e número de sementes por kg foram realizadas em balança de precisão com três casas decimais, seguindo as recomendações das Regras para Análise de Sementes (BRASIL, 2009), sendo o a análise dos resultados realizada através da estatística descritiva.

O estudo foi conduzido no Laboratório de Botânica da Faculdade Dom Luiz de Orleans e estado da Bahia.

\section{RESULTADOS E DISCUSSÃO}

As sementes de Byrsonima verbascifolia apresentaram uma média de $0,83 \mathrm{~cm}$ de comprimento, $0,9 \mathrm{~cm}$ de largura, $0,88 \mathrm{~cm}$ de espessura, e peso de $0,66 \mathrm{~g}$, peso de mil sementes de $335,47 \mathrm{~g}$ e 2.981,26 sementes por $\mathrm{kg}$, conforme apresentado na Tabela 1. O parâmetro de maior desvio foi verificado na largura e espessura, com 0,072 e 0,070 respectivamente, sendo os mesmo que apresentaram o maior coeficiente de variação de $8,00 \%$ e $10,61 \%$, respectivamente. Bragança, no município de Ribeira do Pombal, 
Em relação à distribuição dos resultados biométricos através de classes, constata-se que para o comprimento as sementes possuem grande maioria dos valores de 0,75 a $0,9 \mathrm{~cm}$, com destaque à maior concentração na classe de 0,80 a $0,85 \mathrm{~cm}$, com menor quantidade de sementes na maior classe que varia de 0,95 a $1,00 \mathrm{~cm}$ (Figura 1).

Para a largura, observa-se uma maior concentração nas classes 0,86 a $1,00 \mathrm{~cm}$, com maior representação da classe de $0,86-0,93 \mathrm{~cm}$.
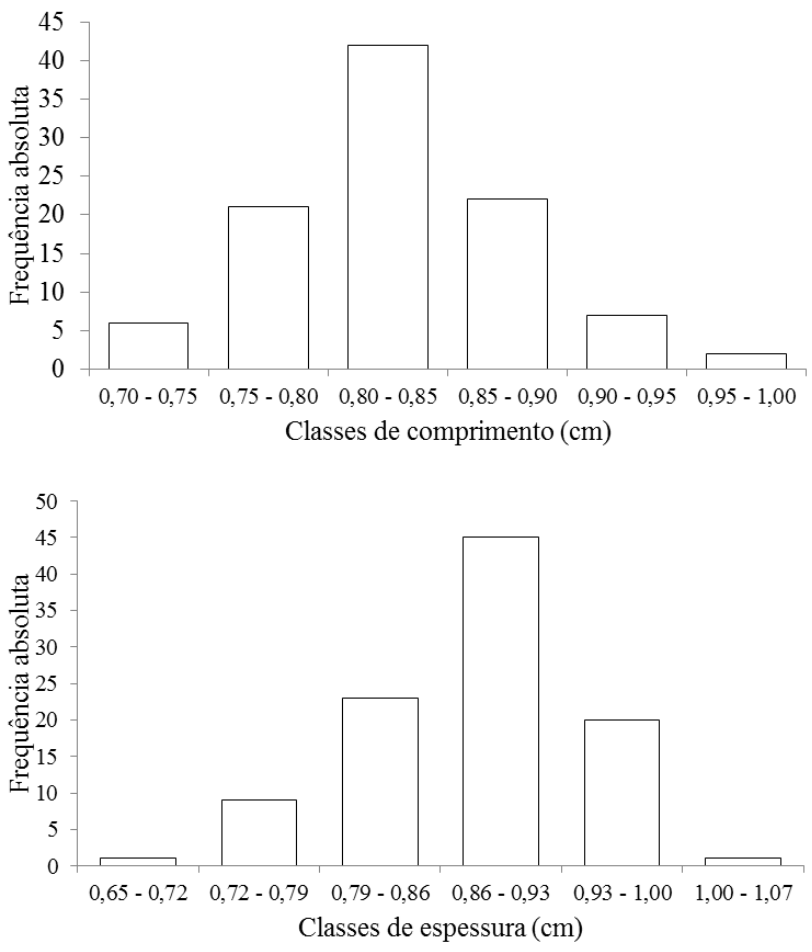

A espessura teve a mesma classe de maior número de sementes iguais a da largura, porém com uma distribuição normal mais simétrica.

Já para o parâmetro peso, nota-se que a maioria das sementes possui de 0,27 a $0,33 \mathrm{~g}$, apresentando significativa quantidade de sementes nos maiores pesos.
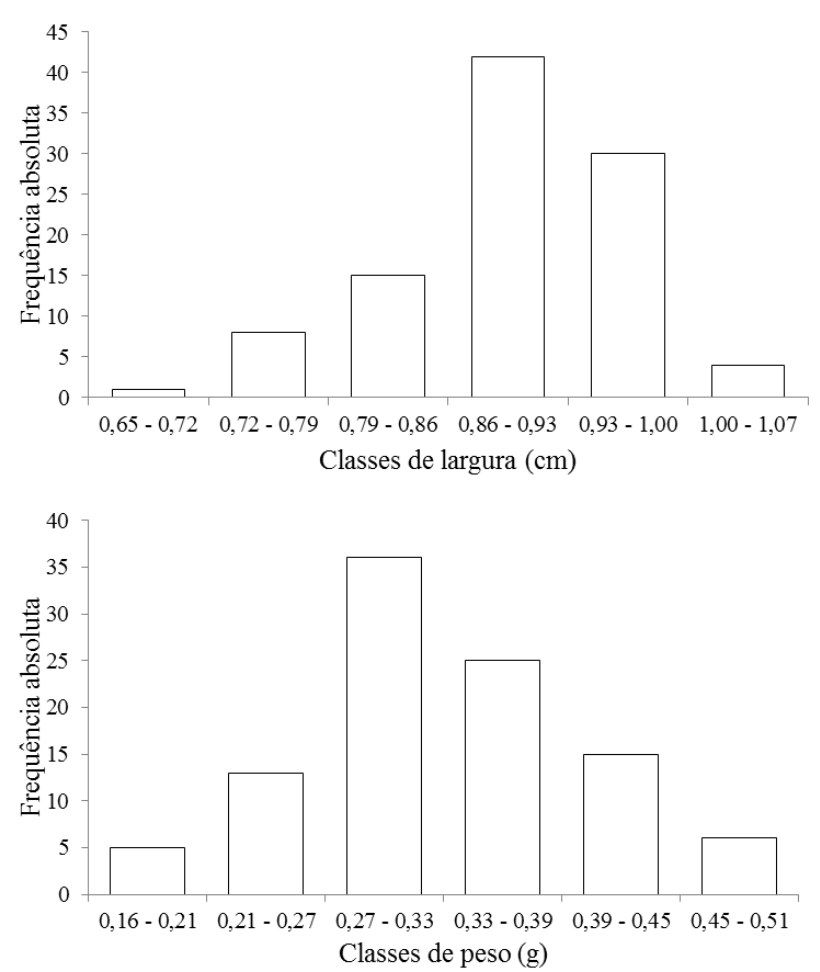

Figura 1. Classes de distribuição de comprimento $(\mathrm{cm})$, largura $(\mathrm{cm})$, espessura $(\mathrm{cm})$ e peso $(\mathrm{g})$ de sementes de Byrsonima verbascifolia (L.) DC., provenientes da região semiárida da Bahia.

Gusmão et al. (2006) analisando a espécie Byrsonima verbascifolia (L.) DC. coletada em um remanescente de cerrado em Minas Gerais observaram que a maioria dos frutos apresentou massa de matéria fresca entre $0,27 \mathrm{~g} \mathrm{e} 0,62 \mathrm{~g}(36 \%)$ e entre $1,31 \mathrm{~g}$ e $2,00 \mathrm{~g}$ (32\%), comprimento entre 8,4 $\mathrm{mm}$ e $9,5 \mathrm{~mm}(27 \%)$ e entre $10,6 \mathrm{~mm}$ e $12,8 \mathrm{~mm}$ (29\%), e diâmetro entre 9,2 mm e $10,7 \mathrm{~mm}(31 \%)$ e entre $13,6 \mathrm{~mm}$ e $15 \mathrm{~mm}(23 \%)$. Em relação à biometria do endocarpo (endocarpo + semente), os mesmos autores constaram que massa de matéria fresca, comprimento e diâmetro mais frequentes entre, respectivamente, $0,16 \mathrm{~g}$ e $0,25 \mathrm{~g}, 5,8 \mathrm{~mm}$ e 6,5 $\mathrm{mm}$ e $6,6 \mathrm{~mm}$ e $7,4 \mathrm{~mm}$, valores de parâmetros menores que os vistos no presente estudo.

Araújo et al. (2009) observaram em sementes obtidas no litoral do município de Maceió, Alagoas, um peso de mil sementes muito menor $(136,30 \mathrm{~g})$ ao encontrado no presente estudo $(335,47 \mathrm{~g})$, conferindo consequentemente um maior número de sementes por quilo (3.244). Os autores caracterizaram ainda a porcentagem de polpa e sementes dos frutos, com, respectivamente, $81,64 \%$ e $18,36 \%$, com um rendimento de polpa de $63,04 \%$, sendo segundo Guimarães e Silva (2008) um alimento rico em fibras, com destaque ao seu valor nutricional, servindo ainda para contribuir com a agregação de valor deste produto regional e a comercialização deste fruto.

As diferenças entre os resultados dos autores citados com os comparados no presente estudo podem estar associados às influências climáticas e edáficas de cada região (GUSMÃO et al., 2006), uma vez que as expressões de determinadas características podem estar relacionadas ao ambiente as quais as espécies vegetais estão sujeitas, como o 
índice de pluviosidade, variações de temperatura e comprimento do dia, o que acaba por ressaltar aspectos específicos de sua composição genética que não se manifestariam em outro local (BOTEZZELI et al., 2000).

\section{CONCLUSÕES}

$\mathrm{O}$ estudo permitiu analisar biometricamente as sementes de Byrsonima verbascifolia (L.) DC., contribuindo para o conhecimento das características físicas das sementes desta espécie encontrada na região semiárida do estado da Bahia.

\section{REFERÊNCIAS}

ANDRADE, L. I. F. de; LIMA, J. S. S. de; OLIVEIRA, K. J. B. de; SOARES, A. P. da S.; SILVA, J. N. da. Agropecuária Científica no Semiárido, Campina Grande, v. 12, n. 1, p. 73-82, 2016.

ARAÚJO, R. R. de; SANTOS, E. D. dos; LEMOS, E. E. P. de; ALVES, R. E. Caracterização biométrica de frutos e sementes de genótipos de murici (Byrsonima verbascifolia (L.) Rich.) do tabuleiro costeiro de Alagoas. Revista Caatinga, Mossoró, v. 22, n. 3, p. 220-224, 2009.

ARAÚJO, A. M. S.; TORRES, S. B.; NOGUEIRA, N. W.; FREITAS, R. M. O. de; CARVALHO, S. M. C. Caracterização morfométrica e germinação de sementes de Macroptilium martii benth. (Fabaceae). Revista Caatinga, Mossoró, v. 27, n. 3, p. 124-131, 2014.

ARAÚJO, B. de A.; SILVA, M. C. B. da; MOREIRA, F. J. C.; SILVA, K. da F.; TAVARES, M. K. das N. Caracterização biométrica de frutos e sementes, química e rendimento de polpa de juazeiro (Ziziphus joazeiro Mart.). Agropecuária Científica no Semiárido, Campina Grande, v. 11, n. 2, p. 15-21, 2015.

ASSIS, J. P. de; LINHARES, P. C. F.; LIMA, G. K. L. de; PEREIRA, M. F. S.; SOUSA, R. P. de; PAIVA, A. C. C. de. Análise biométrica de sementes de feijão bravo (Capparis flexuosa) planta medicinal em Mossoró-RN. Agropecuária Científica no Semiárido, Campina Grande, v. 9, n. 1, p. 94-98, 2013.

BOTEZELLI, L.; DAVIDE, A. C.; MALAVASI, M. M. Características dos frutos e sementes de quatro procedências de Dipteryx alata Vogel (Baru). Cerne, Viçosa, v. 6, p. 9-18, 2000.

BRAZ, M. do S. S.; SOUZA, V. C. de; ANDRADE, L. A. de; BRUNO, R. de L. A.; OLIVEIRA, L. S. B. de; SILVA, J. M. Caracterização morfológica de frutos, sementes e plântulas de jacarandá-dabahia (Dalbergia nigra (Vell.) Fr. All.ex. Benth) LeguminosaePapilonoidea. Revista Brasileira de Ciências Agrárias, Recife, v.4, n.1, p.67-71, 2009.

COSTA, F. R. da; RÊGO, E. R. do; RÊGO, M. M. do; NEDER, D. G.; SILVA, S. de M.; SCHUNEMANN, A.
P. P. Análise biométrica de frutos de umbuzeiro do semiárido brasileiro. Bioscience Journal, Uberlândia, v. 31, n. 3, p. 682-690, 2015.

DANTAS, B. F.; MATIAS, J. R.; MENDES, R. B.; RIBEIRO, R. C. "As sementes da Caatinga são...": um levantamento das características das sementes da Caatinga. Informativo ABRATES, Londrina, v. 24, n. 3, p. 18-23, 2014.

DINIZ, F. O.; MEDEIROS FILHO, S.; BEZERRA, A. M. E.; MOREIRA, F. J. C. Biometria e morfologia da semente e plântula de oiticica. Revista Verde de Agroecologia e Desenvolvimento Sustentável, Pombal, v. $10, \quad$ n. 2 , p. 183-187, 2015. DOI: http://dx.doi.org/10.18378/rvads.v10i2.2965.

Flora do Brasil 2020. Programa Reflora. Jardim Botânico do Rio de Janeiro. Disponível em: <http://floradobrasil.jbrj.gov.br/>. Acesso em: 06 jan. 2018.

GUIMARÃES, M. M.; SILVA, M. S. Valor nutricional e características químicas e físicas de frutos de murici-passa (Byrsonima verbascifolia).

Ciência e Tecnologia de Alimentos, v. 28, n. 4, p. 817 821, 2008.

GUSMÃO, E.; VIEIRA, F. de A.; FONSECA JÚNIOR, E. M. da. Biometria de frutos e endocarpos de murici (Byrsonima verbascifolia Rich. ex A. Juss.). Cerne, Viçosa, v. 12, n. 1, p. 84-91, 2006.

INEMA. Instituto do Meio Ambiente e Recursos Hídricos. CBH Itapicuru. Disponível em: <http://www.inema.ba.gov.br/gestao-2/comites-debacias/comites/cbh-itapicuru/>. Acesso em: 01 jun. 2017.

LEÃO, N. V. M.; FELIPE, S. H. S.; SHIMIZU, E. S. C.; SANTOS FILHO, B. G. dos; KATO, O. R.; BENCHIMOL, R. L. Biometria e diversidade de temperaturas e substratos para a viabilidade de sementes de ipê amarelo. Informativo ABRATES, Londrina, v. 25, n.1, p. 50-54, 2015.

LOUREIRO, M. B.; TELES, C. A. S.; VIRGENS, I. O.; ARAÚJO, B. R. N. de; FERNANDEZ, L. G.; CASTRO, R. D. de. Aspectos morfoanatômicos e fisiológicos de sementes e plântulas de Amburana cearensis (FR. ALL.) A.C. Smith (Leguminosae - Papilionoideae). Revista Árvore, Viçosa, v. 37, n. 4, p. 679-689, 2013.

LUCENA, E. O. De; LÚCIO, A. M. F. da; BAKKE, I. A.; PIMENTA, M. A. C.; RAMOS, T. M. Biometria e qualidade fisiologica de sementes de juazeiro (Ziziphus joazeiro Marth.) de diferentes matrizes do semiarido paraibano. Agropecuária Científica no Semiárido, Campina Grande, v. 13, n. 4, p. 275-280, 2017.

ROVERI NETO, A.; PAULA, R. C. de. Variabilidade entre árvores matrizes de Ceiba speciosa St. Hil para características de frutos e sementes. Revista Ciência 
Agronômica, Fortaleza, v. 48, n. 2, p. 318-327, 2017. DOI: http://dx.doi.org/10.5935/1806-6690.20170037.

SILVA, R. M. da; RIBEIRO, R. de T. M.; COUTINHO, D. J. G.; SILVA, S. Í. da; GALLÃO, M. I. Caracterização de frutos, sementes, plântulas e germinação de Jeniparana. Ceres, Viçosa, v. 61, n.5, p. 746-751, 2014a. DOI: http://dx.doi.org/10.1590/0034-737X201461050019.

SILVA, A. C. da; SANTOS, J. F.; d'ARÊDE, L. O.; MORAIS, O. M.; COSTA, E. M.; SILVA, E. A. A. da. Caracterização biométrica e superação de dormência em sementes de Chloroleucon foliolosum (Benth.) G. P. Lewis. Revista Brasileira de Ciências Agrárias, Recife, v. $9, \quad$ n. $4, \quad$ p. 577-582, 2014b. DOI:10.5039/agraria.v9i4a4586.
SILVA, R. M. da; CARDOSO, A. D.; DUTRA, F. V.; MORAIS, O. M. Aspectos biométricos de frutos e sementes de Caesalpinia ferrea Mart. ex Tul. provenientes do semiárido baiano. Revista de Agricultura Neotropical, Cassilândia, v. 4, n. 3, p. 8591, 2017. 